\title{
QSurface: fast identification of surface expression markers in cancers
}

\author{
Yourae Hong ${ }^{\dagger}$, Choa Park ${ }^{\dagger}$, Nayoung Kim², Juyeon $\mathrm{Cho}^{2}$, Sung Ung Moon ${ }^{1}$, Jongmin Kim² , Euna Jeong ${ }^{*}$ \\ and Sukjoon Yoon ${ }^{1,2^{*}}$
}

From The 28th International Conference on Genome Informatics

Seoul, Korea. 31 October - 3 November 2017

\begin{abstract}
Background: Cell surface proteins have provided useful targets and biomarkers for advanced cancer therapies. The recent clinical success of antibody-drug conjugates (ADCs) highlights the importance of finding selective surface antigens for given cancer subtypes. We thus attempted to develop stand-alone software for the analysis of the cell surface transcriptome of patient cancer samples and to prioritize lineage- and/or mutation-specific over-expression markers in cancer cells.

Results: A total of 519 genes were selected as surface proteins, and their expression was profiled in 14 cancer subtypes using patient sample transcriptome data. Lineage/mutation-oriented analysis was used to identify subtype-specific surface markers with statistical confidence. Experimental validation confirmed the unique over-expression of predicted surface markers (MUC4, MSLN, and SLC7A11) in lung cancer cells at the protein level. The differential cell surface gene expression of cell lines may differ from that of tissue samples due to the absence of the tumor microenvironment.

Conclusions: In the present study, advanced 3D models of lung cell lines successfully reproduced the predicted patterns, demonstrating the physiological relevance of cell line-based 3D models in validating surface markers from patient tumor data. Also QSurface software is freely available at http://compbio.sookmyung.ac.kr/ qsurface.
\end{abstract}

Keywords: Cancer transcriptome, Cancer mutations, Antibody-drug conjugates, Software development

\section{Background}

Cell surface proteins have provided major targets and biomarkers for anticancer therapies. In colorectal cancer, the expression of surface proteins such as $\mathrm{CDH} 17$, CD138 and members of the integrin family is related with tumor progression [1]. Another surface protein, SEZ6L2 was identified a novel prognostic marker in non-small cell lung cancer (NSCLC) [2]. Epidermal growth factor receptor (EGFR) is over-expressed cell types and plays a key role in cancer progression. Indeed, many drugs targeting EGFR have been developed [3]. In

\footnotetext{
*Correspondence: eajeong@sookmyung.ac.kr; yoonsj@sookmyung.ac.kr ${ }^{\dagger}$ Equal contributors

${ }^{1}$ Center for Advanced Bioinformatics \& Systems medicine, Department of Biological Sciences, Sookmyung Women's University, Hyochangwon-gil 52, Yongsan-gu, Seoul 140-742, Republic of Korea

Full list of author information is available at the end of the article
}

addition, HER2, a plasma membrane-bound protein and member of the ErbB family, is significantly overexpressed in $10-15 \%$ of breast cancers, referred to HER2-positive breast cancer [4,5].

More recently, cell surface proteins have been successfully used as targets for antibody-drug conjugates (ADC) as part of cancer therapy [6-8]. ADCs are composed of antibodies for targeting and cytotoxic drugs and linker proteins for attaching to and cleaving the target. Once ADCs reach and attach to the target antigen on the cancer cell surface, receptor-mediated endocytosis internalizes the antibody and cytotoxic drug. Thus, surface antigens for ADCs should exhibit tumor-specific expression for the selective targeting of ADCs, and facilitate receptor-mediated endocytosis. A complete list of tumor-specific cell surface markers will help identify potential antigens for this type of advanced therapy. 
The Cancer Genome Atlas (TCGA) is one of the largest datasets from pan-cancer analyses [9]. The released multiomics dataset includes genome, transcriptome and proteome data for tissue samples from thousands of cancer patients, covering 30 cancer types. The proteome dataset, generated using reverse-phase protein arrays (RPPA), is limited in the availability of specific antibodies [10]. For TCGA, the expression data for a few hundred proteins are available [11]. However, TCGA transcriptome data includes the expression profiles of $\sim 20,000$ genes, enabling the identification of selectively overexpressed genes corresponding to surface proteins [12].

As an analysis tool to find differentially expressed genes, cBioPortal $[13,14]$ is useful but has limitation of comparative analyses using two or more omics datasets. For example, cBioPortal doesn't provide extensive analyses using both somatic mutation and gene expression datasets. In addition, cell surface genes and proteins are not classified in cBioPortal.

In the present study, we developed stand-alone software, QSurface, to analyze lineage- and/or mutation-specific cell surface transcriptome marker from cancer patients' samples obtained from TCGA. Selected expression markers were validated at the protein level using lung adenocarcinoma (LUAD) cell lines. Notably, the gene expression of extracellular/membrane proteins exhibits inconsistent patterns between cell lines and patient tissue samples [15]. In the present study, we attempted to overcome this problem using advanced 3D sphere-based assays which provided a physiologically relevant microenvironment for the tested cell lines [16]. The present software and assay method will provide fast and efficient tools to identify novel tumorspecific cell surface markers for advanced cancer therapies such as ADCs.

\section{Method}

\section{Data acquisition}

RNA sequencing version 2 (RNASeqV2) data from patients' tumor and normal tissue samples were downloaded from TCGA website (http://cancergenome.nih.gov/) in 2015. The RNASeqV2 data were sequenced using the Illumina HiSeq 2000 and Illumina Genome Analyzer (GA) platforms. We selected 658 tumor samples with matched normal samples obtained from the same patient in 14 cancer types, satisfying the requirement more than 10 samples (Additional file 1: Table S1). The expression level of each gene was normalized using RNA-Seq by Expectation Maximization (RSEM) count estimates method and we converted the data to the $\log 2$ scale. For breast invasive carcinoma (BRCA), two tumor patients were duplicated using primary and metastatic samples. We excluded two metastasis samples.

TCGA provides multi-dimensional datasets, which means that one samples has genotype and expression data together. We obtained the somatic mutation dataset from cBioPortal. The curated dataset has been processed from published literature. Somatic mutations are curated and annotated with information of variant effects, predicted from SIFT [17] and Polyphen-2 [18] algorithms. To analyze only non-synonymous mutations including truncating mutation and deleterious missense mutation, we excluded neutral mutations predicted from two algorithms. The criteria of non-synonymous mutations are SIFT score $<0.05$ or Polyphen-2 score $>0.85$. Gene expression data were integrated with these processed mutation data. After annotating tumor samples, 555 tumor samples are remained for analysis (Additional file 1: Table S1).

\section{Selection of cell surface genes}

We selected 'cell surface' (Gene Ontology term GO:0009986) from the cellular components category to identify cell surface genes from the AmiGO website, October 2016 [19, 20]. A total of 524 genes belonging to the 'cell surface' category were located in the external part of the cell wall or plasma membrane. Among of these genes, 519 genes remained for analysis after mapping using the TCGA RNASeqV2 data.

\section{Statistical analysis}

To identify differentially expressed cell surface genes, we used $\log 2$ delta and t-test $P$-values. We calculated $\log 2$ delta as the average difference in the expression levels for lineage-specific cell surface genes between tumor and normal samples and for mutation-specific cell surface genes between mutant and wild-type tumor samples per lineage.

\section{$2 D$ cell culture}

Three types of cell lines, an STK11 mutant type (A549, H460, H23, and H1993), STK11 wild type (H522, H322M, HCC-827, and H1975), and STK11-recoverd type (A549STK11, H460-STK11, H23-STK11, and H1993-STK11) were used for validation experiments. HCC-827 and H1975 cells were obtained from the American Type Culture Collection (ATCC, Manassas, VA, USA), respectively. All other STK11 mutant and wild-type cell lines were obtained from the National Institutes of Health, National Cancer Institute (NCI, Frederick, MD, USA). STK11 mutant cell lines and wild-type cell lines were cultured in RPMI 1640 (HyClone Laboratories, Logan) supplemented with $10 \%$ fetal bovine serum (HyClone Laboratories) and $1 \%$ antibiotics (GIBCO BRL, Thermo Fisher Scientific). STK11-recoverd cell lines were cultured in the same medium with added $1 \mu \mathrm{g} / \mathrm{ml}$ puromycine. A total of $1 \sim 3 \times$ $10^{5}$ cells per well were seeding on a 6 well culture plate for monolayer cell culture during 5 days. 


\section{$3 D$ cell culture}

Cancer stem-like cell (CSLC) spheres were cultured in serum-free conditioned DMEM/F-12 medium supplemented with $20-\mathrm{ng} / \mathrm{ml}$ EGF, 20-ng/ml basic fibroblast growth factor, and B27 (Thermo Fisher Scientific). The cells were maintained in a humidified atmosphere of $5 \%$ $\mathrm{CO}_{2}$ and $95 \%$ air at $37{ }^{\circ} \mathrm{C}$ and the culture medium was refreshed every 2 to 3 days. The culture plates for stemlike cells (SLCs) were coated with a $5-\mathrm{mg} / \mathrm{ml}$ solution of poly-2-hydroxyethyl methacrylate (Sigma-Aldrich) in 95\% ethanol. The same amount of cells as 2D cell culture was seeded in a 6-well plate for sphere culture.

\section{Western blot}

Total cell extracts were prepared by incubating the cells in lysis buffer (RIPA Cell lysis buffer containing $150 \mathrm{mM}$ sodium chloride, $1 \%$ Triton X-100, 1\% sodium deoxycholate, $0.1 \%$ SDS, $50 \mathrm{mM}$ Tris- $\mathrm{HCl}$, pH 7.5, and $2 \mathrm{mM}$ EDTA, sterile solution, GenDEPOT) on ice for $30 \mathrm{~min}$. Cell debris was removed by centrifugation, and the total protein levels in the supernatants were quantified using the Bradford method (Bio-Rad). Equal amounts of protein $(50 \mu \mathrm{g})$ were heated at $95{ }^{\circ} \mathrm{C}$ for $5 \mathrm{~min}$, electrophoretically resolved using 12\% SDS-PAGE, and then transferred to nitrocellulose membranes (Millipore). The membranes were blocked with TBST [20 mM Tris- $\mathrm{HCl}, \mathrm{pH} 7.6$, and $0.1 \%$ Tween-20] containing 5\% skim milk for $1 \mathrm{~h}$ and then hybridized as indicated to specific primary antibodies
(1:1000 dilution) at $4{ }^{\circ} \mathrm{C}$ overnight. The membranes were washed and hybridized to HRP-conjugated secondary antibodies for $1 \mathrm{~h}$ at room temperature. Specific bands were visualized using an enhanced chemiluminescence (ECL) detection system (Thermo, Logan, UT, USA) and an LA3000 luminescence image analyzer (Fujifilm, Tokyo, Japan). Antibodies against MUC4 and SLC7A11 were purchased from Abcam PLC. Antibodies against Mesothelin and GAPDH were purchased from Cell Signaling. GAPDH was used as a loading control. The anti-STK11 antibody was purchased from Santa Cruz.

\section{Results and discussion Implementation of QSurface}

We implemented QSurface, a tool for exploring lineageand/or mutation-specific gene expression of all potential surface proteins (Fig. 1a). Genes for surface proteins were defined using the category information in Gene Ontology database [20].

Users can browse differential gene expression of 29 known target antigens that are currently under phase I or II clinical trials [6, 21, 22] (Additional file 1: Table S2). Candidate genes are separated into two groups, overexpressed in diverse tumors and over-expressed in specific tumors.

To identify lineage-specific gene expression, users can search differentially expressed genes by selecting a specific lineage, for example, lung adenocarcinoma tumor
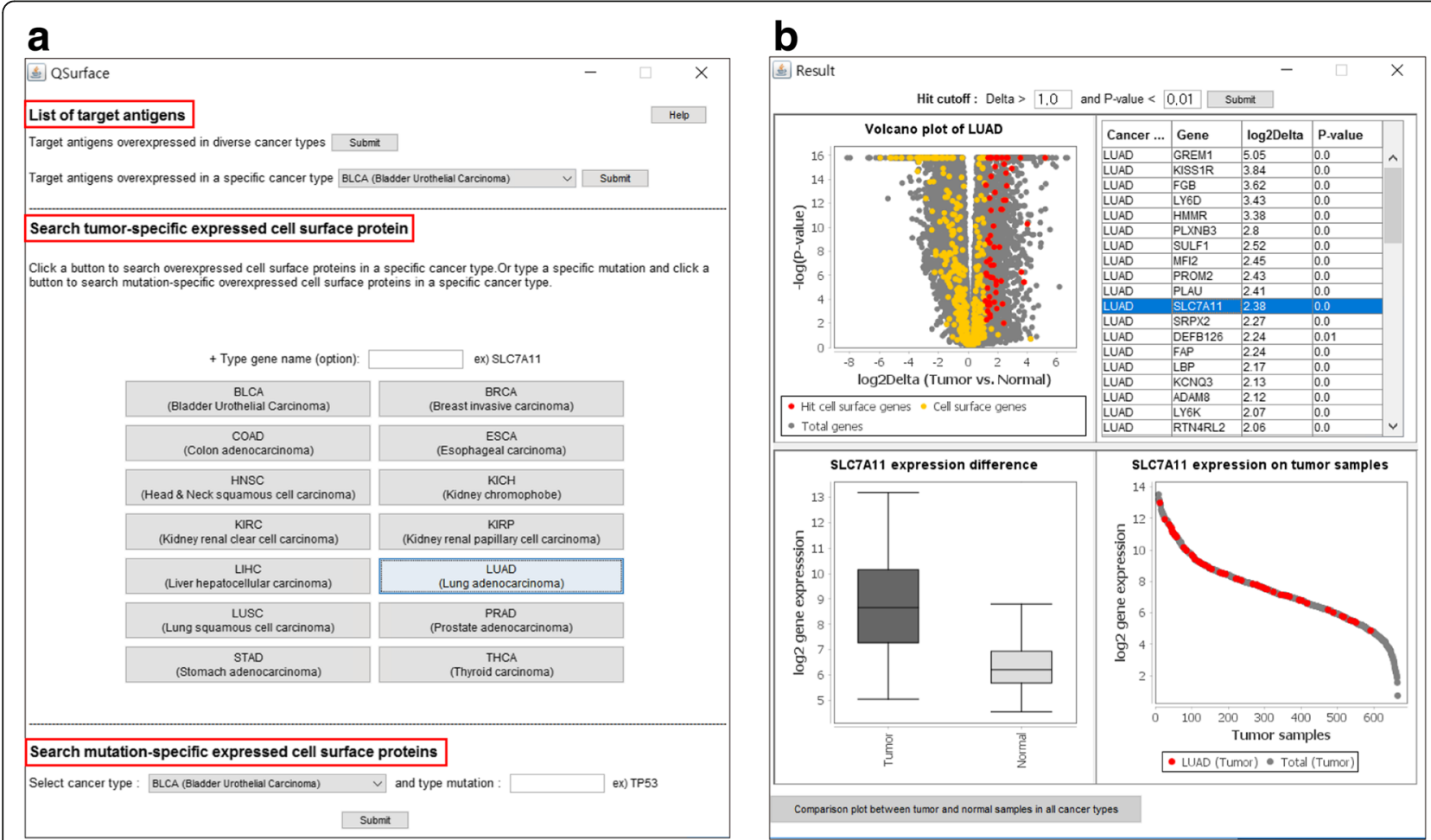

Fig. 1 Overview of QSurface. a Graphical user interface of QSurface front page. b Snapshot of lineage-oriented profiling for lung adenocarcinoma (LUAD) 


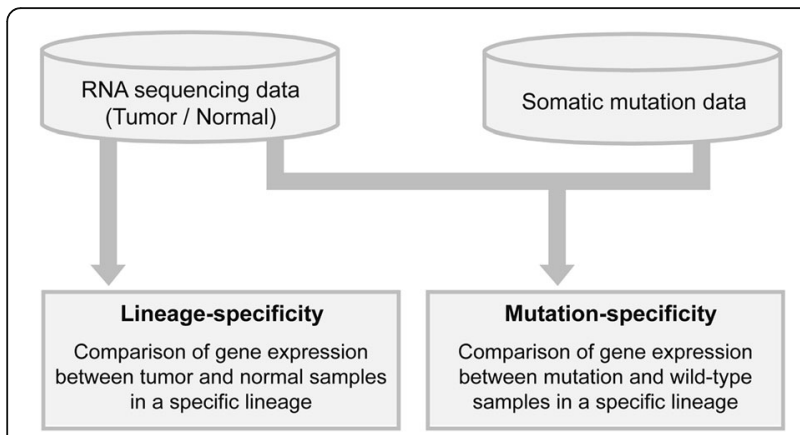

Fig. 2 Dataflow and data processing of QSurface. Lineage-oriented profiling uses paired tumor and normal samples in RNA sequencing data, and mutation-oriented profiling uses somatic mutation data and only tumor samples in RNA sequencing data

LUAD (Fig. 1a). A volcano plot is used to display the expression differences between tumor and normal samples. Firstly, a total of 20,531 genes and 519 cell surface genes are visualized in grey and yellow colors, respectively. The significant differentially expressed genes are shown in red color in the plot and listed as a table after submitting criteria $(\log 2$ Delta $>1.0$ and $P$-value $<0.01)$ in the top of the window (Fig. 1b upper). By clicking a hit gene (SLC7A11) from the list, the box plot and the waterfall plot will be popped up to show the expression pattern between tumor and normal samples and the lineage specificity (in this case LUAD) among all samples for the selected hit gene SLC7A11 (Fig. 1b bottom). Lastly, the comparison of gene expression pattern between tumor and normal samples among all cancer types is available for the hit gene by clicking "Comparison plot" in the bottom of the window.

Furthermore, mutation-specific hits can be also displayed by adding mutation criteria together with lineage information. Users can obtain over-expressed hits enriched in mutant samples over wild type samples for a given lineage. The overall data processing and analytical flow are described in Fig. 2.

To summarize, QSurface is a tool to analyze lineageand/or mutation-specific gene expression of all potential surface proteins between tumor and normal samples or

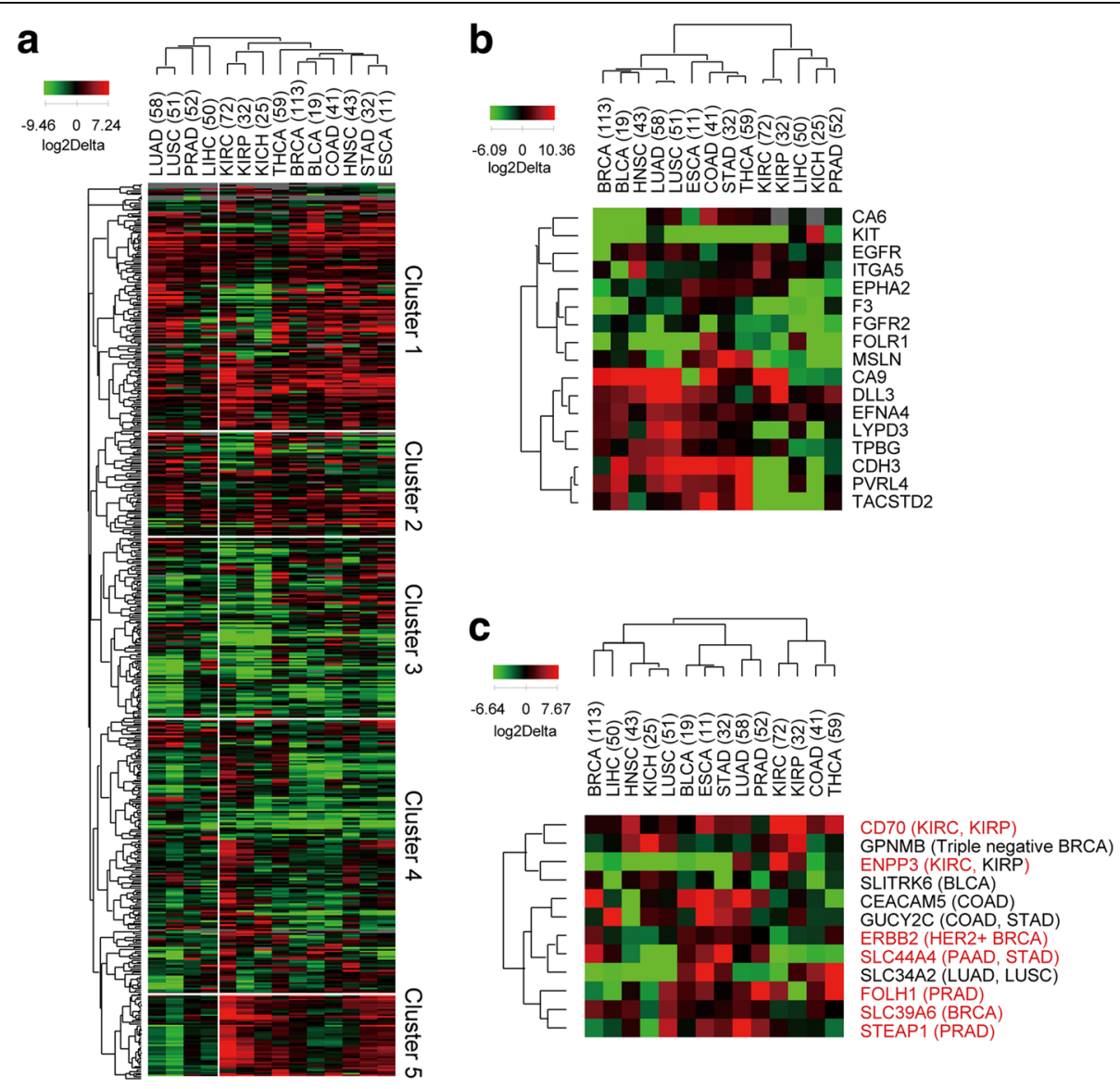

Fig. 3 Hierarchical clustering of cell surface genes and known antigens for ADCs. a A heatmap of 519 cell surface genes and 14 cancer types. Heatmaps of ADC target genes differentially expressed on the diverse cancer types in (b) and other ADC target differentially expressed on the specific cancer types in (c). Cancer types are described in parentheness and significantly overexpressed target genes (log2Delta $>1$ and $p$ value $<0.01)$ in the specific cancer type are shown in red. QCanvas was used to cluster and draw heatmaps [32] 

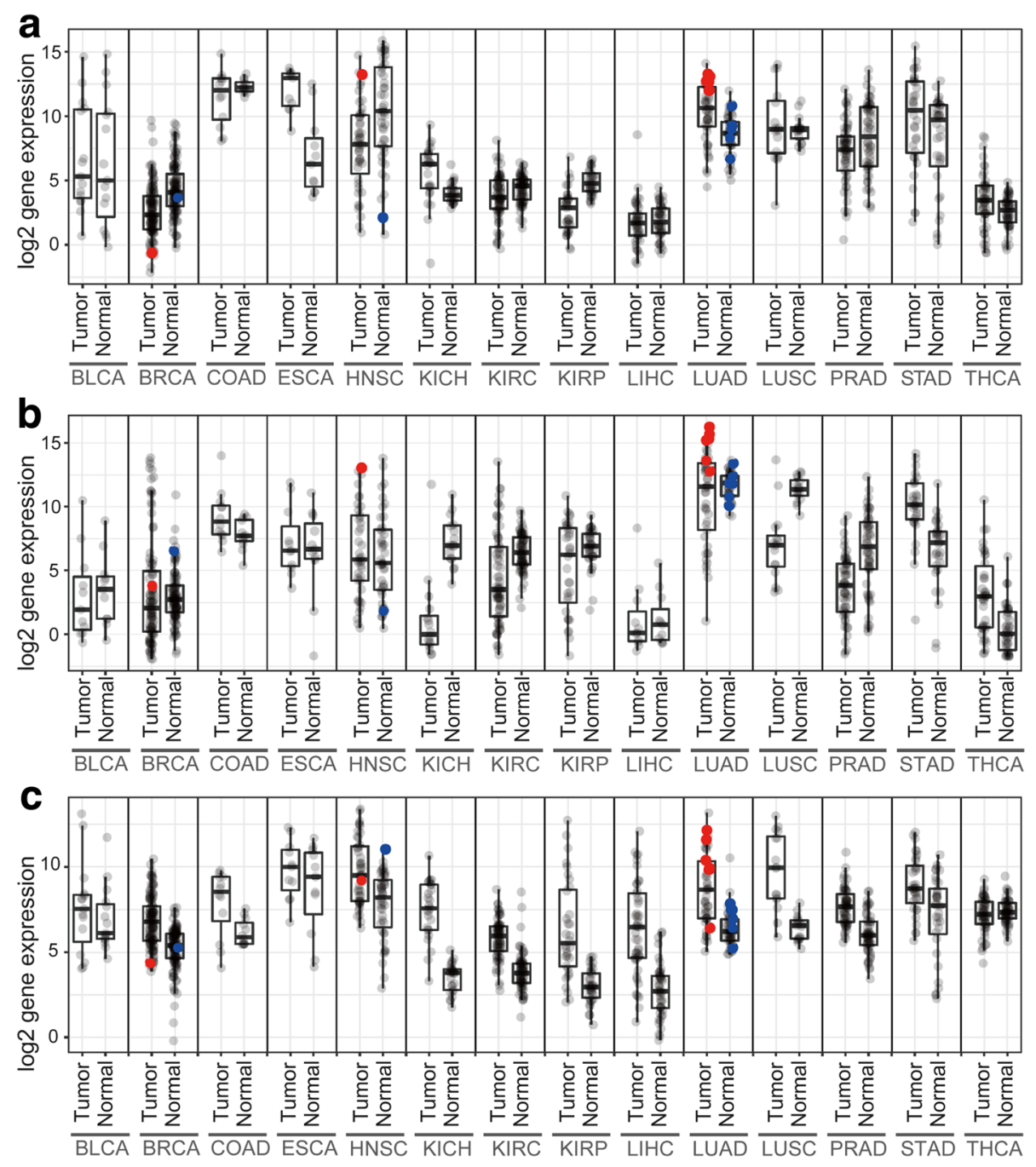

Fig. 4 Comparison of MUC4 (a), MSLN (b) and SLC7A11 (c) expression profiles in 14 cancer types. Patient tumor samples with STK11 mutation and the corresponding normal samples are colored in red and blue, respectively. The number of patient samples with STK11 mutation BRCA $=1$, $\mathrm{HNSC}=1$ and $\mathrm{LUAD}=6$

mutant and wild-type samples. It is helpful to find potential cell surface marker using difference of gene expression with statistical confidence. QSurface is a stand-alone Java tool that can be run on any operating system. JavaStat and JFreeChart library packages were used to calculate Student's t-test and draw all plots, respectively.

\section{Lineage-based analysis of cell surface genes and known target antigens}

We analyzed the profile of 519 cell surface genes in 14 cancer lineages to show how many genes are over-expressed on diverse tumor samples or specific tumor samples via normal samples. The differentially expressed genes were classified into 5 groups (Fig. 3a). The genes Cluster 1 and 2 were over-expressed in multiple diverse lineages, while those in Cluster 5 were over-expressed in a subset of lineages and down-regulated in lung, prostate and liver cancer types. However, the genes belonging to Clusters 3 and 4 were relatively down-regulated in tumors compared to normal samples. Many of the genes in Cluster 4 were uniquely overexpressed in the kidney cancer type (KIRC). The lineage-wide distribution of all genes and 519 cell surface genes, and potential cell surface maker genes is shown in Additional file 1: Fig. S1.

Some of target antigens for ADCs are known to be expressed on tumor and normal tissue [23]. For example, target antigens over-expressed on specific cancer type, SLC34A2, translating NaPi2b, have high expressed in normal patients [24]. In case of RCC, membrane EGFR was expressed higher than tumor samples via normal samples. But the expression of cytoplasmic EGFR protein is lower than normal samples [25]. As described in Section 3.1, a total of 29 known target antigens of ADCs were analyzed 
in Fig. $3 \mathrm{~b}$ and c. The 17 target antigens of ADCs which were known as over-expressed on diverse tumors, are actually classified into two groups (Fig. $3 \mathrm{~b}$ ). The upper cluster (CA6, KIT, EGFR, ITGA5, EPHA2, F3, FGFR2, FOLR1 and MSLN) exhibited lineage-dependent, limited expression patterns, while the bottom cluster (CA9, DDL3, EFNA4, LYPD3, TPBG, CDH3, PVRL4, and TACSTD2) is over-expressed on diverse tumors. The other 12 antigens of ADCs which were known as over-expressed on specific tumors, showed the over-expression on diverse lineages (Fig. 3c). Our analysis confirmed that seven genes (CD70, ENPP3, ERBB2, SLC44A4, FOLH1, SLC39A6 and STEAP1) were significantly over-expressed in the kn0own target lineages (in red), except ENPP3 in KIRP with $\log 2$ Delta 1.06 and $P$-value 0.03 .

\section{Identification of mutation-specific expression of surface genes}

We analyzed the mutation-oriented profiles of gene expression in diverse lineages using QSurface. For example, the genes MUC4, MSLN, and SLC7A11 were predicted as STK11 mutation-specific cell surface markers in lung cancer samples (Fig. 4). Although MUC4 is not annotated using a 'cell surface' GO term, this transmembrane glycoprotein is differentially expressed on diverse cancer cell types, including LUAD [26, 27]. In the present study, MUC4 over-expression was observed in LUAD tumors compared to normal samples. Interestingly, this overexpression was highly associated with STK11 mutation in LUAD samples $(\log 2$ Delta $=2.76, P$-value $=0.002) \quad($ Fig. 4a). MSLN (or Mesothelin) is a known target gene over-expressed by the cells of solid tumors, particularly mesothelioma and LUAD [28]. MSLN-targeted ADC candidates are currently under investigation in phase I/II clinical trials for diverse cancer types. In the present analysis, we observed that MSLN expression was selectively associated with STK11 mutant samples $(\log 2$ Delta $=4.74$, P-value $=9 . \mathrm{E}-04)($ Fig. 4b). Lastly, SLC7A11 is classified as a cell surface GO term, and this gene is known to be highly expressed by colon, kidney, and liver cancer cells [29]. Mutation-oriented analysis also revealed the strong association of SLC7A11 expression with STK11 mutations. The present mutant-oriented analysis of surface markers improved the statistical confidence in the selectivity of the expression of these genes in diverse cancer lineages. In the present study, MUC4, MSLN, and SLC7A11 showed high $\log 2$ Delta values of $2.76,4.74$, and 1.55 , with $P$-values of 0.002 , 9.e-04, and 0.04, respectively.

\section{Validation of surface markers with advanced 3D assays} Cancer cell culture system are classified into twodimensional (2D) and three-dimensional (3D) cancer models [30]. Compared to a typical 2D monolayer cancer model, the 3D model mimics the in vivo environment because solid tumors grow in three-dimensions creating a unique microenvironment and facilitating cell-cell communication [31]. We measured the protein expression of the three surface markers (MUC4, MSLN and SLC7A11) in lung cell lines under 2D and 3D culture conditions. In the $2 \mathrm{D}$ system, the expression showed no difference between the STK11 mutant and wild-type cell lines of LUAD (Fig. 5a, Additional file 1: Fig. S2). However, the protein level of MUC4, MSLN, and SLC7A11 were significantly increased in STK11 mutant cell lines (Fig. 5b), confirming the transcriptionlevel prediction of QSurface from patient samples. This result demonstrates the physiological relevance of the 3D sphere model for reproducing the expression feature
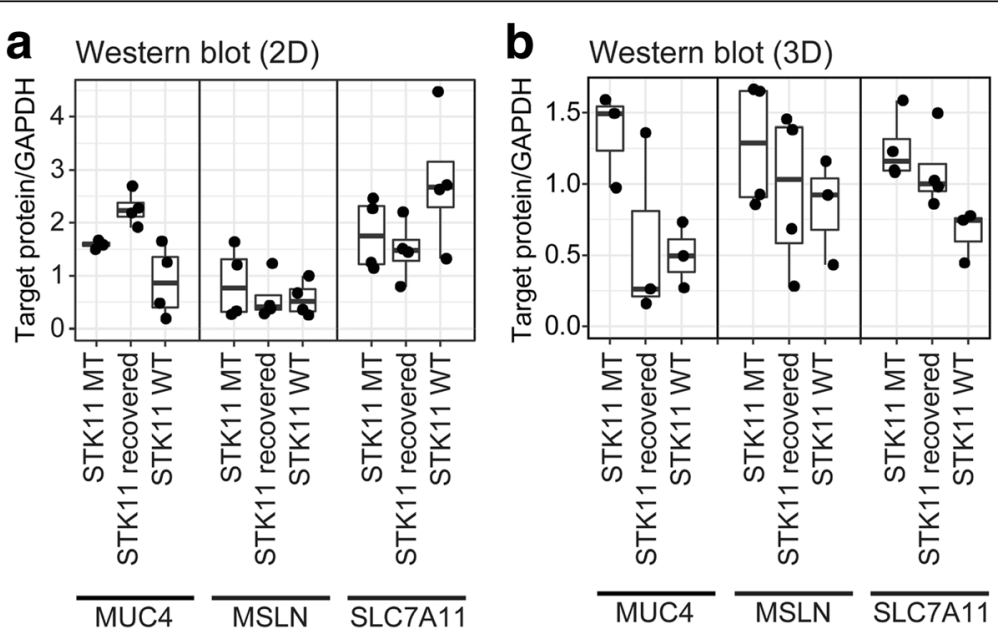

Fig. 5 Comparison of MUC4, MSLN, SLC7A11 protein expression among STK11 mutant, recovered, and wild-type cell lines in 2D and 3D culture models. (a) P-values in 2D culture model are 0.04, 0.23, and 0.11 for MUC4, MSLN, and SLC7A11 resp. (b) P-values in 3D culture model are 0.01, 0.11 , and 0.008 for the same order 
of surface markers identified or predicted from patient samples. This validation confirms that QSurface provides useful and reliable tools for identifying mutation/ lineage-specific surface markers and/or target antigens for ADCs.

\section{Conclusions}

This study presents QSurface, rapid and efficient tools to identify novel tumor-specific cell surface markers for advanced cancer therapies. QSurface provides two analyzing method, lineage- $/$ and mutation-oriented profiles. To identify potential surface genes, QProfile used fold changes to find sensitivity of gene expression on given conditions. By using QSurface, we obtained 3 STK11-mutant specific expression markers, MUC4, MSLN, and SLC7A11 in LUAD. Furthermore, advanced 3D cell line models of lung cancer successfully reproduced the predict patterns by QSurface. And it demonstrates the physiological relevance of cell line-based 3D models with patient tumor data and confirms that QSurface is useful and reliable tools for identifying mutation/lienage-specific cell surface markers.

\section{Additional files}

Additional file 1: Table S1. Data description of TCGA RNA sequencing data. Table S2. List of antibody-drug conjugates. Figure S1. Distribution of tumor sample-specific gene expression in 14 cancer types. Totally 20,531 genes, 519 cell surface marker, and significant cell surface hits (log2Delta $>1$ and $p$-value $<0.01$ ) are illustrated in grey, yellow, and red, respectively. Figure S2. Western blot analysis of the MUC 4, MSLN and SLC7A11 expression on STK11 mutant, restored and wild type cell lines. (A) gene expression on STK11 mutant, restored and wild-type cell lines on 2D culture status and (B) on 3D culture status. (DOCX $1902 \mathrm{~kb}$ )

\section{Abbreviations}

2D: Two-Dimensional; 3D: Three-Dimensional; ADC: Antibody-drug Conjugates; BRCA: Breast Invasive Carcinoma; CSLC: Cancer Stem-like Cell; GO: Gene Ontology; LUAD: Lung Adenocarcinoma; RSEM: RNA-Seq by Expectation Maximization; SLC: Stem-like Cell; TCGA: The Cancer Genome Atlas

\section{Acknowledgements}

The authors would like to thank Zhijun Luo (Department of Biochemistry, Boston University School of Medicine) for providing the STK11 plasmid.

\section{Funding}

The publication cost of this article was funded by grants from the National Research Foundation of Korea (KRF), including the Science Research Center Program (NRF-2016R1A5A1011974), the Bio \& Medical Technology Development Program (NRF-2012M3A9B6055398), and the Basic Science Research Program (NRF-2015R1D1A1A01057815 and NRF-

2016R1D1A1B03935110), funded by the Korean government (MEST). Also, this Research was supported by the Sookmyung Women's University BK21 Plus Scholarship.

\section{Availability of data and materials}

The RNA sequencing dataset analyzed during the current study are available in the TCGA repository, http://cancergenome.nih.gov/. Also Somatic mutation dataset analyze during the current study are available in the cBioPortal repository, http://www.cbioportal.org [13, 14]. Lastly, the Gene Ontology dataset are available in the Gene Ontology Consortium, http:// www.geneontology.org/ [19, 20].

\section{About this supplement}

This article has been published as part of BMC Systems Biology Volume 12 Supplement 2, 2018: Proceedings of the 28th International Conference on Genome Informatics: systems biology. The full contents of the supplement are available online at https://bmcsystbiol.biomedcentral.com/articles/ supplements/volume-12-supplement-2.

\section{Authors' contributions}

YH performed construction of QSurface, analyzing cell surface data, and prepared the article. CP performed 2D and 3D cell modeling experiments, interpreted experiment result and prepared the article; NK performed analyzing cell surface data. EJ, SM and JK provided scientific direction. YH, $C P, E J$ and SY wrote this article. SY designed and coordinated the overall research and experiments; and all authors discussed the results and commented on the article. All authors read and approved the final manuscript.

\section{Ethics approval and consent to participate}

Not applicable.

\section{Consent for publication}

Not applicable.

\section{Competing interests}

Not applicable.

\section{Publisher's Note}

Springer Nature remains neutral with regard to jurisdictional claims in published maps and institutional affiliations.

\section{Author details}

${ }^{1}$ Center for Advanced Bioinformatics \& Systems medicine, Department of Biological Sciences, Sookmyung Women's University, Hyochangwon-gil 52, Yongsan-gu, Seoul 140-742, Republic of Korea. ${ }^{2}$ Department of Biological Sciences, Sookmyung Women's University, Seoul 140-742, Republic of Korea.

Published: 19 March 2018

\section{References}

1. Luque-Garcia JL, Martinez-Torrecuadrada JL, Epifano C, Canamero M, Babel I, Casal $\mathrm{J}$, et al. Differential protein expression on the cell surface of colorectal cancer cells associated to tumor metastasis. Proteomics. 2010;10(5):940-52.

2. Ishikawa N, Daigo Y, Takano A, Taniwaki M, Kato T, Tanaka S, Yasui W, Takeshima Y, Inai K, Nishimura H, et al. Characterization of SEZ6L2 cellsurface protein as a novel prognostic marker for lung cancer. Cancer Sci. 2006:97(8):737-45.

3. Seshacharyulu P, Ponnusamy MP, Haridas D, Jain M, Ganti AK, Batra SK, et al. Targeting the EGFR signaling pathway in cancer therapy. Expert Opin Ther Targets. 2012;16(1):15-31.

4. Malhotra GK, Zhao X, Band H, Band V, et al. Histological, molecular and functional subtypes of breast cancers. Cancer Biol Ther. 2010;10(10):955-60.

5. Incorvati JA, Shah S, Mu Y, Lu J, et al. Targeted therapy for HER2 positive breast cancer. J Hematol Oncol. 2013;6:38.

6. Diamantis N, Banerji $\mathrm{U}$, et al. Antibody-drug conjugates-an emerging class of cancer treatment. Br J Cancer. 2016:114(4):362-7.

7. Panowski S, Bhakta S, Raab H, Polakis P, Junutula JR, et al. Site-specific antibody drug conjugates for cancer therapy. MAbs. 2014;6(1):34-45.

8. Thomas A, Teicher BA, Hassan R, et al. Antibody-drug conjugates for cancer therapy. Lan Oncol. 2016:17(6):e254-62.

9. Cancer Genome Atlas Research N, Weinstein JN, Collisson EA, Mills GB, Shaw KR, Ozenberger BA, Ellrott K, Shmulevich I, Sander C, Stuart JM, et al. The cancer genome atlas pan-cancer analysis project. Nat Genet. 2013;45(10): 1113-20.

10. Spurrier B, Ramalingam S, Nishizuka S, et al. Reverse-phase protein lysate microarrays for cell signaling analysis. Nat Protoc. 2008;3(11):1796-808.

11. Li J, Lu Y, Akbani R, Ju Z, Roebuck PL, Liu W, Yang JY, Broom BM, Verhaak $R G$, Kane DW, et al. TCPA: a resource for cancer functional proteomics data. Nat Methods. 2013;10(11):1046-7.

12. Peng L, Bian XW, Li DK, Xu C, Wang GM, Xia QY, Xiong Q, et al. Large-scale RNA-Seq transcriptome analysis of 4043 cancers and 548 normal tissue controls across 12 TCGA cancer types. Sci Rep. 2015;5:13413. 
13. Cerami E, Gao J, Dogrusoz U, Gross BE, Sumer SO, Aksoy BA, Jacobsen A, Byrne CJ, Heuer ML, Larsson E, et al. The cBio cancer genomics portal: an open platform for exploring multidimensional cancer genomics data. Cancer Discov. 2012:2(5):401-4.

14. Gao J, Aksoy BA, Dogrusoz U, Dresdner G, Gross B, Sumer SO, Sun Y, Jacobsen $A$, Sinha R, Larsson $E$, et al. Integrative analysis of complex cancer genomics and clinical profiles using the cBioPortal. Sci Signal. 2013;6(269): pl1.

15. Hong Y, Kim N, Li C, Jeong E, Yoon S, et al. Patient sample-oriented analysis of gene expression highlights extracellular signatures in breast cancer progression. Biochem Biophys Res Commun. 2017;487(2):307-12.

16. Edmondson R, Broglie JJ, Adcock AF, Yang L, et al. Three-dimensional cell culture systems and their applications in drug discovery and cell-based biosensors. Assay Drug Dev Technol. 2014;12(4):207-18.

17. Sim NL, Kumar P, Hu J, Henikoff S, Schneider G, Ng PC, et al. SIFT web server: predicting effects of amino acid substitutions on proteins. Nucleic Acids Res. 2012;40(Web Server issue:W452-7.

18. Adzhubei IA, Schmidt S, Peshkin L, Ramensky VE, Gerasimova A, Bork P, Kondrashov AS, Sunyaev SR, et al. A method and server for predicting damaging missense mutations. Nat Methods. 2010;7(4):248-9.

19. Ashburner M, Ball CA, Blake JA, Botstein D, Butler H, Cherry JM, Davis AP, Dolinski K, Dwight SS, Eppig JT, et al. Gene ontology: tool for the unification of biology. The gene ontology consortium. Nat Genet. 2000;25(1):25-9.

20. Gene Ontology C, et al. Gene ontology consortium: going forward. Nucleic Acids Res. 2015:43(Database issue):D1049-56.

21. Polakis $\mathrm{P}$, et al. Antibody drug conjugates for cancer therapy. Pharmacol Rev. 2016;68(1):3-19.

22. Goldenberg DM, Cardillo TM, Govindan SV, Rossi EA, Sharkey RM, et al. Trop2 is a novel target for solid cancer therapy with sacituzumab govitecan (IMMU-132), an antibody-drug conjugate (ADC). Oncotarget. 2015;6(26): 22496-512.

23. Teicher BA, Chari RV, et al. Antibody conjugate therapeutics: challenges and potential. Clinical cancer research : an official of the American Association for Cancer Research. 2011;17(20):6389-97.

24. Lin K, Rubinfeld B, Zhang C, Firestein R, Harstad E, Roth L, Tsai SP, Schutten M, Xu K, Hristopoulos M, et al. Preclinical development of an anti-NaPi2b (SLC34A2) antibody-drug conjugate as a therapeutic for non-small cell lung and ovarian cancers. Clinical cancer research : an official J of the American Association for Cancer Research. 2015;21(22):5139-50.

25. Pu YS, Huang CY, Kuo YZ, Kang WY, Liu GY, Huang AM, Yu HJ, Lai MK, Huang SP, Wu WJ, et al. Characterization of membranous and cytoplasmic EGFR expression in human normal renal cortex and renal cell carcinoma. J Biomed Sci. 2009;16:82.

26. Carraway KL, Theodoropoulos G, Kozloski GA, Carothers Carraway CA, et al. Muc4/MUC4 functions and regulation in cancer. Future Oncol. 2009;5(10): 1631-40.

27. Rokutan-Kurata M, Yoshizawa A, Sumiyoshi S, Sonobe M, Menju T, Momose M, Koyama M, Shigeto S, Fujimoto M, Zhang M, et al. Lung adenocarcinoma with MUC4 expression is associated with smoking status, HER2 protein expression, and poor prognosis: Clinicopathologic analysis of 338 cases. Clin Lung Cancer. 2017;18(4):e273-81.

28. Morello A, Sadelain M, Adusumilli PS, et al. Mesothelin-targeted CARs: driving T cells to solid tumors. Cancer Discov. 2016;6(2):133-46.

29. Jiang L, Kon N, Li T, Wang SJ, Su T, Hibshoosh H, Baer R, Gu W, et al. Ferroptosis as a p53-mediated activity during tumour suppression. Nature. 2015;520(7545):57-62.

30. Pampaloni F, Reynaud EG, Stelzer EH, et al. The third dimension bridges the gap between cell culture and live tissue. Nat Rev Mol Cell Biol. 2007;8(10): 839-45.

31. Breslin $S$, O'Driscoll $L$, et al. The relevance of using $3 D$ cell cultures, in addition to 2D monolayer cultures, when evaluating breast cancer drug sensitivity and resistance. Oncotarget. 2016;7(29):45745-56.

32. Kim N, Park H, He N, Lee HY, Yoon S, et al. QCanvas: an advanced tool for data clustering and visualization of genomics data. Genomics Inform. 2012; 10(4):263-5.

\section{Submit your next manuscript to BioMed Central and we will help you at every step:}

- We accept pre-submission inquiries

- Our selector tool helps you to find the most relevant journal

- We provide round the clock customer support

- Convenient online submission

- Thorough peer review

- Inclusion in PubMed and all major indexing services

- Maximum visibility for your research

Submit your manuscript at www.biomedcentral.com/submit

Biomed Central 